\title{
Anderson 型杂多酸作为催化剂在有机合成中的应用
}

\author{
魏哲宇 $a$ 常亚林 $a$ 余焓*, $a, b$ 韩生*, $a$ 永革 $*, b$ \\ ( ${ }^{a}$ 上海应用技术大学化学与环境工程学院 上海 201418) \\ ( ${ }^{b}$ 清华大学化学系 北京 100084)
}

\begin{abstract}
摘要 Anderson 型杂多酸 (通式为 $\left[\mathrm{XM}_{6} \mathrm{O}_{24}\right]^{n-}$ )是一类具有特殊结构和性质的多金属-氧簇无机化合物, 由于其分子结 构易于化学修饰和可设计性以及其在材料、催化和医药等领域具有很高的应用价值, 因此一直受到研究者们的广泛关 注. 近几年来, Anderson 型杂多酸在催化有机氧化反应中的应用对于绿色催化过程的研究逐渐显露出非常重要的意义. 本综述对 Anderson 型杂多酸在有机合成反应中的催化应用进行了相关的调研，根据 Anderson 型多金属氧酸盐的结构 分类对这些工作进行了归纳概述与总结. 这将为 Anderson 型杂多酸的催化应用以及为绿色催化的研究提供新的思路. 关键词 杂多酸; Anderson 结构; 有机合成; 分子催化; 绿色化学
\end{abstract}

\section{Application of Anderson Type Heteropoly Acids as Catalysts in Organic Synthesis}

\author{
Wei, Zheyu $^{a} \quad$ Chang, Yalin $^{a} \quad$ Yu, Han*,a,b Han, Sheng*,a $\quad$ Wei, Yongge*,b \\ ( ${ }^{a}$ College of Chemistry and Environmental Engineering, Shanghai Institute of Technology, Shanghai 201418) \\ ( ${ }^{b}$ Department of Chemistry, Tsinghua University, Beijing 100084)
}

\begin{abstract}
Anderson type heteropoly acids, also known as Anderson type polyoxometalates, are a kind of important structures in polyoxometalates. Their general structural formula can be expressed as $\left[\mathrm{XM}_{6} \mathrm{O}_{24}\right]^{n-}$, in which the core heteroatom $\mathrm{X}$ can almost be replaced by almost any metal or nonmetal element in the periodic table. Due to unique structure easy to be modified with organic ligands and designability, as well as their potential applications in materials, catalysis and medicines, Anderson type heteropoly acids have been widely concerned by researchers. In recent years, the application of Anderson type heteropoly acids in organic synthesis has gradually shown great significance for the study of green catalytic process. In this paper, the catalytic application of Anderson type heteropoly acids in organic synthesis has been reviewed and summarized according to the structure classification of Anderson type polyoxometalates. This will be helpful for the researchers to further study the catalytic application of Anderson heteropoly acids and provides new ideas for the research of green catalysis.
\end{abstract}

Keywords heteropoly acids; Anderson structure; organic synthesis; molecular catalysis; green chemistry

\section{1 引言}

Anderson 型杂多酸, 又称 Anderson 型多金属氧酸 盐, 是多金属氧族化合物中的一类重要结构, 结构通式 可表示为 $\left[\mathrm{XM}_{6} \mathrm{O}_{24}\right]^{n-}$ 或 $\left[\mathrm{H}_{x}\left(\mathrm{XO}_{6}\right) \mathrm{M}_{6} \mathrm{O}_{18}\right]^{n-}(\mathrm{M}=\mathrm{Mo}$ 或 W), 其中心杂原子 $\mathrm{X}$ 几乎可以换成元素周期表中任意的金 属或非金属原子, 且 Anderson 型杂多酸稳定的构型为 平面环状结构, 如图 $1 \mathrm{a}$ 和 $1 \mathrm{~b}$. Anderson 型杂多酸表面 $\mathrm{O}$ 原子存在 3 种不同类型的配位模式, 因而多酸表面不同 位点的反应活性具有较大差异. 此外, 多酸表面 $\mathrm{O}$ 原子 的质子化程度也存在明显差异, 因而可分为下述 2 种 (图 1c): 一种是 Anderson 型杂多酸表面 $\mu_{3}-\mathrm{O}$ 末质子化 的(称之为 $\mathrm{A}$ 型); 另一种则是 $\mu_{3}-\mathrm{O}$ 质子化的(称之为 $\mathrm{B}$ 型). 如果中心杂原子价态较高(氧化态 $>4$ ), Anderson 型 杂多酸还发现以类似七钼酸根的折叠异构体构型存在
(图 $1 \mathrm{~d}$ 和 $1 \mathrm{e}$ ), 对于低价态的中心金属原子, 这种结构则 需要有机配体保护才能稳定存在(图 1f $)^{[1-5]}$.

从结构上来看, Anderson 型杂多酸的氧原子以 6 个 共边的八面体围绕中心杂原子形成一个具有共平面性 的环状结构或折叠的扭曲结构, 其中心杂原子变化多样 且易于被化学修饰, 因此该结构具有较高的配位活性, 通过对其进行化学修饰可以使其结构与性质更加丰富.

经典的杂多酸结构大多为纳米或亚纳米尺寸且粒 子均一, Anderson 型杂多酸的八面体连接方式以及易于 被修饰等特性更是决定其成为一类很好的亚纳米建筑 单元，设计合成出具有各种特殊尺寸和性质的有机一无 机多酸化合物在现代材料、医药、催化等领域有着非常 重要的应用价值.

对于 Anderson 型杂多酸的早期研究主要集中在新 化合物的合成制备与表征等方面，国内王恩波课题组、

\footnotetext{
*E-mail: hanyu0220@tsinghua.edu.cn; hansheng654321@sina.com; yonggewei@tsinghua.edu.cn

Received May 26, 2020; published June 29, 2020.

Project supported by the National Natural Science Foundation of China (Nos. 21971134, 21631007, 21225103).

项目受国家自然科学基金(Nos. 21971134, 21631007, 21225103)资助.
} 


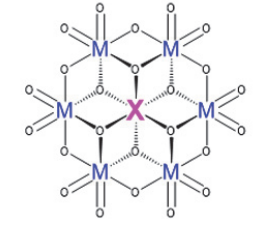

(a)

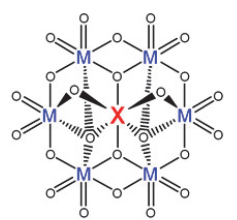

(d)

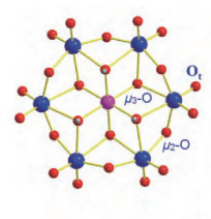

(b)

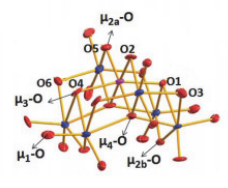

(e)

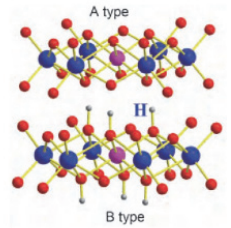

(c)

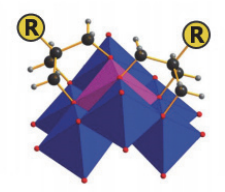

(f)
图 1 (a) $\alpha$-Anderson 型杂多酸结构示意图; (b, c) $\alpha$-Anderson 型杂多酸 球棍模型图; (d) $\beta$-Anderson 杂多酸结构示意图; (e) $\beta$-Anderson 型杂多 酸球棍模型图; (f) 有机配体稳定的 $\beta$-Anderson 型杂多酸

Figure 1 (a) Structure diagram of $\alpha$-Anderson type heteropoly acids; (b, c) model diagram of $\alpha$-Anderson type heteropoly acids Club; (d) structure diagram of $\beta$-Anderson type heteropoly acids; (e) model diagram of $\beta$-Anderson type heteropoly acids Club; (f) organically modified $\beta$-Anderson type heteropoly acids

魏永革课题组、周百斌课题组、牛景杨课题组、王秀丽 课题组和国外的 Kreb 等课题组在这一领域作出了较为 突出的贡献.

2014 年, 魏哲宇等 ${ }^{[6]}$ 发明了一种液相扩散单晶生长 加样法以及专用加样管, 该发明可以显著提高培养单晶 的工作效率, 通过该方法培养的单晶质量也有显著的提 升. 魏永革课题组通过该方法培养了大量不同类型的 Anderson 型杂多酸及其有机衍生物的晶体(图 2), 并通 过单晶 X-射线衍射、红外光谱、核磁共振谱和液相色谱 等方法确定了许多新型 Anderson 型杂多酸及其有机衍 生物的晶体结构, 极大地推动了 Anderson 型杂多酸的 合成与结构化学的发展.

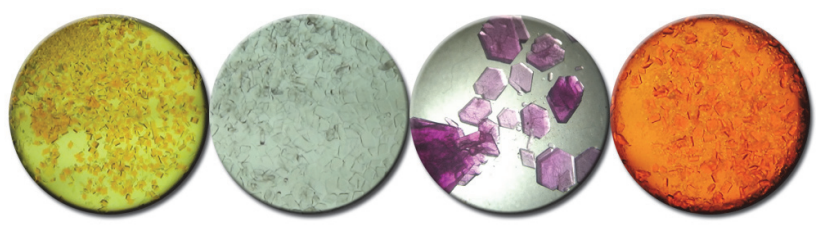

图 2 一种液相扩散专用加样管 ${ }^{[6]}$ 制备的几种 Anderson 型杂多酸晶体 Figure 2 Several Anderson type heteropoly acids crystals prepared by the special sample tube for liquid phase diffusion technique ${ }^{[6]}$

随着 Anderson 型杂多酸的结构发展, 越来越多的 研究者们开始对 Anderson 型杂多酸的可控修饰以及催 化应用进行了深入的研究. 他们发现 Anderson 型杂多 酸不但具有酸碱特性, 而且因其中心原子的差异还具有 丰富的氧化还原性, 由于其结构稳定性好, 可作均相及 非均相反应, 甚至还可以通过适当的化学修饰后作相转 移催化剂或离子液体催化剂, 因此, Anderson 型杂多酸 是一种具有很高催化活性的新型亚纳米级分子, Ander- son 型杂多酸的材料化和功能化研究也逐渐成为多酸化 学中的一个前沿热门领域 ${ }^{[7-12]}$. 但 Anderson 型杂多酸的 催化应用早期的工作主要集中在氧化脱硫和工业废水 的处理 ${ }^{[13-17]}$, 而在有机合成中的应用很少涉及.

人类社会的进步离不开有机合成化学的发展, 然而 在大多数有机合成反应中, 不仅所用到的催化剂昂贵、 反应条件严苛危险，而且反应所生成的副产物往往对环 境会造成一定程度的污染, 因此, 用化学的技术和方法 减少或停止那些对人类生态环境有害的物质产生, 不仅 是有机合成反应中的难点, 也是当今绿色化学研究的重 点, 更是 21 世纪化学发展的重要方向之一 ${ }^{[18-21]}$. 随着 Anderson 型杂多酸的催化应用进展, 研究者们发现: 在 有机合成反应中, 用 Anderson 型多金属氧酸盐及其衍 生物来代替传统的金属或非金属催化剂进行催化氧化, 不仅催化效率高且催化过程中所产生的副产物少, 正是 一种对环境友好的绿色高效催化新路线.

在这些工作报道中所合成使用的 Anderson 型杂多 酸催化剂种类繁多, 为了更好地对这些研究工作进行归 纳与概述, 本综述根据近年来关于 Anderson 型杂多酸 的有机催化应用的相关文献报道，以 Anderson 型多金 属氧酸盐的阴离子结构为分类依据, 大致将这些工作分 为两大类(图 3), 同时为了方便描述, 我们对以下分类定 义了相关的字母简写: (1)简单的 Anderson 型杂多酸(以 下简写为 P) 的催化应用; (2) Anderson 型杂多酸有机衍 生物(以下简写为 $\mathrm{PO}$ )的催化应用; 在这两大类中再根 据 Anderson 型杂多酸的阳离子形式将催化剂细分为两 小类: (1)简单无机阳离子(如 $\mathrm{NH}_{4}^{+} 、 \mathrm{Na}^{+} 、 \mathrm{H}^{+} 、 \mathrm{~K}^{+}$, 以 下简写为 $\left[\mathrm{I}^{+}\right]^{+}$)的 Anderson 型杂多酸; (2)有机阳离子(以 下简写为 $\left.[\mathrm{O}]^{+}\right)$的 Anderson 型杂多酸.

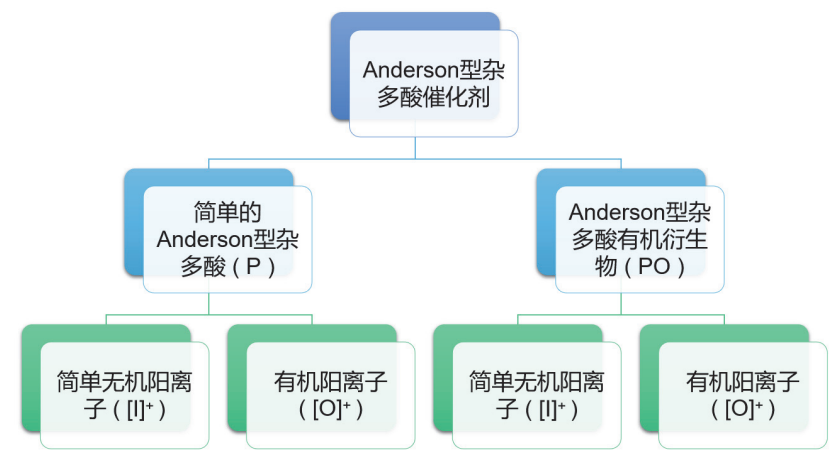

图 3 本文对 Anderson 型杂多酸的树状结构分类图

Figure 3 Tree-structure classification of Anderson type heteropoly acids in this paper

\section{Anderson 型杂多酸在有机合成中的催化应 用}

\subsection{Anderson 型杂多酸 $(\mathrm{P})$ 的催化}

\subsection{1 [I $]^{+} \mathrm{P}$}

2016 年, Kadam 和 Gokavi ${ }^{[22]}$ 对 Anderson 型铬(III) 
钼酸盐催化氧化乙酸肼的动力学和催化机理进行了相 关研究, 该反应催化剂最初由 $\mathrm{H}\left[\mathrm{Cr}^{\mathrm{III}}(\mathrm{OH})_{6} \mathrm{Mo}_{6} \mathrm{O}_{18}\right]^{2-}$ 氧 化生成为 $\left[\mathrm{O}=\mathrm{Cr}^{\mathrm{V}}(\mathrm{OH})_{6} \mathrm{Mo}_{6} \mathrm{O}_{18}\right]^{3-}$, 然后与酰肼反应生 成目标产物, 在反应过程中不受任何自由基的干预, 这 项工作对于 Anderson 型杂多酸催化氧化酰肼提供了很 好的思路与启发.

2017 年, 余焓等 ${ }^{[23]}$ 提出了一种简单、温和且高效的 需氧氧化胺的方法, 通过无机配体负载的非贵金属催化 剂 $\left(\mathrm{NH}_{4}\right)_{n}\left[\mathrm{MMo}_{6} \mathrm{O}_{18}(\mathrm{OH})_{6}\right]\left(\mathrm{M}=\mathrm{Cu}^{2+}, \mathrm{Fe}^{3+}, \mathrm{Co}^{3+}, \mathrm{Ni}^{2+}\right.$, $\mathrm{Zn}^{2+} ; n=3$ 或 4)在 $100{ }^{\circ} \mathrm{C}$ 的水中一步合成胺, 证明了通 过改变中心金属原子可以显著提高催化活性和选择性. 在 Anderson 型杂多酸催化剂的存在下, 以 $\mathrm{O}_{2}$ 为唯一氧 化剂, 伯胺和仲胺的催化氧化以及醇和胺的偶联反应可 以顺利地提供各种亚胺, 这种全新的工艺方法开辟了一 条通过无机配体配位金属进行催化氧化的途径.

不久后, 余焓及其合作者 ${ }^{[24]}$ 又提出了一种利用无 机配体负载的 $\mathrm{Cu}$-Anderson 型杂多酸催化剂 $\left(\mathrm{NH}_{4}\right)_{4}-$ $\left[\mathrm{Cu}(\mathrm{OH})_{6} \mathrm{Mo}_{6} \mathrm{O}_{18}\right]$ 在水中好氧氧化醛制备羧酸的方法(图 4). 该方法以氧气为唯一氧化剂, 在极温和的水溶液条 件下进行, 并能适用于多种官能团的醛类化合物. 在该 催化过程中, 铜催化剂 $\left(\mathrm{NH}_{4}\right)_{4}\left[\mathrm{Cu}(\mathrm{OH})_{6} \mathrm{Mo}_{6} \mathrm{O}_{18}\right]$ 可以循 环使用, 研究表明, 连续反应可以进行至少 6 次, 而催 化剂的催化性能均无明显下降. 余焓等提出的这种催化 醛制备羧酸的方法不仅操作简单, 而且避免了使用成本 昂贵、毒性较大的原料, 该催化路线的通用性使其有可 能被工业化应用。

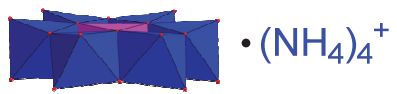

1: $\left(\mathrm{NH}_{4}\right)_{4}\left[\mathrm{CuMo}_{6} \mathrm{O}_{18}(\mathrm{OH})_{6}\right]$

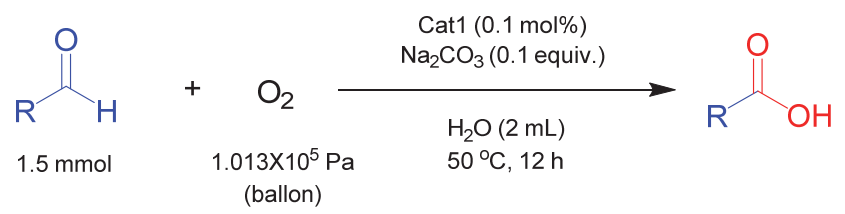

图 4 利用 $\left(\mathrm{NH}_{4}\right)_{4}\left[\mathrm{Cu}(\mathrm{OH})_{6} \mathrm{Mo}_{6} \mathrm{O}_{18}\right]$ 在水中氧化醛制备羧酸的方法路线 Figure 4 A method for preparing carboxylic acids by oxidizing aldehydes in water using $\left(\mathrm{NH}_{4}\right)_{4}\left[\mathrm{Cu}(\mathrm{OH})_{6} \mathrm{Mo}_{6} \mathrm{O}_{18}\right]$

2018 年, 翟永彦等 ${ }^{[25]}$ 利用无机配体负载的 Fe-Anderson 型杂多酸催化剂 $\left(\mathrm{NH}_{4}\right)_{3}\left[\mathrm{Fe}(\mathrm{OH})_{6} \mathrm{Mo}_{6} \mathrm{O}_{18}\right]$, 同样以氧气作为唯一氧化剂, 促进了有机卤化物的好氧 氧化制醛/酮和胺氧化偶联制备亚胺. 该催化剂由廉价 的 $\left(\mathrm{NH}_{4}\right)_{6} \mathrm{Mo}_{7} \mathrm{O}_{24} \bullet 4 \mathrm{H}_{2} \mathrm{O}$ 和 $\mathrm{Fe}_{2}\left(\mathrm{SO}_{4}\right)_{3}$ 合成, 其在卤化物和 胺的催化氧化反应中对多种底物具有非常好的活性, 在 这类催化反应中, 催化剂的催化活性损失很小, 说明无 机配体负载催化剂在催化化学转化中具有很大的潜力, 而且由于该催化剂具有较稳定的无机骨架，从而提供了 良好的稳定性和可重用性, 因此卤化物和胺的催化氧化 可以很容易地提高到克级，具有工业化应用前景.
张梦齐、翟永彦和茹诗等 ${ }^{[26]}$ 报道了一种利用无机配 体负载的碘催化剂 $\left(\mathrm{NH}_{4}\right)_{5}\left[\mathrm{IMo}_{6} \mathrm{O}_{24}\right]$ 好氧氧化醇的有效 方法，该催化体系与多种基团相容，具有较高的选择性 和良好的稳定性. 基于实验结果, 他们提出了碘催化剂 $\left(\mathrm{NH}_{4}\right)_{5}\left[\mathrm{IMo}_{6} \mathrm{O}_{24}\right]$ 催化氧化醇的初步机理(图 5). 其催化 氧化反应与与酶促氧化反应相似, 可分为两个独立的半 反应: $\left(\mathrm{NH}_{4}\right)_{5}\left[\mathrm{I}^{\mathrm{VII}} \mathrm{Mo}_{6}{ }^{\mathrm{VI}} \mathrm{O}_{24}\right]$ 介导的醇氧化反应和 $\left[\mathrm{I}^{\mathrm{V}} \mathrm{Mo}_{6}{ }^{\mathrm{VI}} \mathrm{O}_{24}\right]$ 的二氧偶联氧化反应. 对于无机配体负载 的碘多钼酸催化剂, 在氧化过程中所需要的两个氧化当 量储存在 Anderson 杂多酸骨架的碘中心，同时添加剂 的加入对该反应的进行有着显著的影响, 这很可能是由 于 $\mathrm{Cl}^{-}$或 $\mathrm{Ac}^{-}$作为电子转移介质从而提高了碘多钼酸催 化剂体系的电子转移效率. 这一机理的提出对新型 Anderson 型杂多酸的有机催化氧化的应用发展具有重 要的意义. 这是一种高效、温和的无机配体配位高价碘 催化氧化体系, 该体系具有广泛的底物耐受性、良好的 选择性和可回收性，这种催化系统避免了使用任何有机 配体和有毒氧化剂, 与有机高价碘试剂比较, 更有利于 其在医药、香料和食品添加剂中的应用. 除此以外, $\left(\mathrm{NH}_{4}\right)_{5}-\left[\mathrm{IMo}_{6} \mathrm{O}_{24}\right]$ 的结构也对 Anderson 型杂多酸骨架的 中心离子的替换提供了更多的思路.

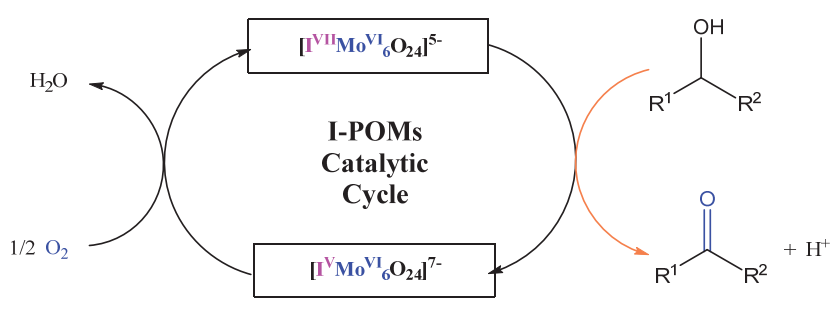

图 5 碘六钼酸催化剂催化醇氧化反应的机理研究 ${ }^{[26]}$

Figure 5 Proposed mechanism for the iodine-POM-catalyzed oxidation of alcohols ${ }^{[26]}$

随后，王静静等 ${ }^{[27]}$ 以 $\mathrm{Zn}$-Anderson 型多金属氧酸盐 $\left(\mathrm{NH}_{4}\right)_{4}\left[\mathrm{Zn}(\mathrm{OH})_{6} \mathrm{Mo}_{6} \mathrm{O}_{18}\right]$ 为催化剂, 建立了一种关于卤化 物与胺的氧化交叉偶联反应、胺的氧化自偶联反应和卤 化物的氧化反应的催化体系(图 6). 在该催化体系的研 究中，他们选择茮胺和氯化苄作为氧化交叉偶联反应的 模型底物, 得到 $N$-芐叉苯丙胺, 收率为 $32 \%$, 选择性为 $47 \%$, 通过对溶剂的篎选得出乙腈为最佳溶剂, 收率为 $89 \%$, 选择性为 $90 \%$. 此外, 缩短和延长反应时间对产 率均有影响, $60{ }^{\circ} \mathrm{C}$ 为最佳反应温度, 最佳催化剂用量为 $1.0 \mathrm{~mol} \%$. 尽管该反应使用单一的氧气作为氧源, 但仍 然可以有效地制备相关的亚胺和醛或酮. 无机配体负载 的锌多酸催化剂不仅易于用水热法制备合成，而且由于 该反应的非均相性, 使得催化剂易于回收. 与铑、钌、 钯等贵金属催化剂相比, 无机配体负载的 Zn-Anderson 型杂多酸催化剂避免了有毒、对空气和水敏感的有机配 体的使用, 因此无机配体修饰杂多酸在有机合成中作为 多相催化剂仍有很大的潜力, 该体系对环境友好且催化 效率较高. 


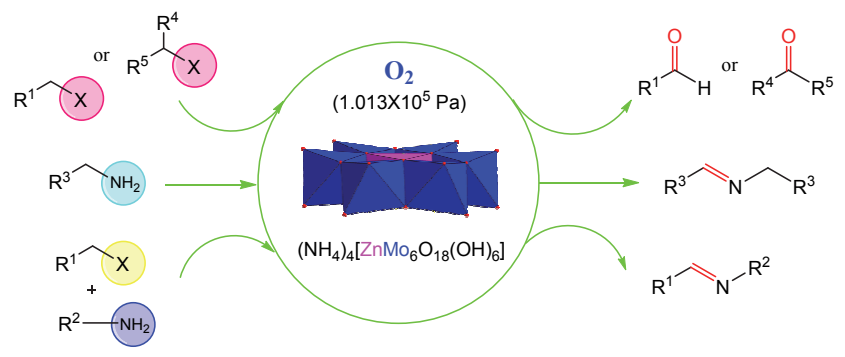

图 6 Zn-Anderson 型杂多酸催化卤化物氧化成醛或酮和胺氧化成亚 胺 ${ }^{[27]}$

Figure 6 Zn-Anderson heteropoly acids catalyzes the oxidation of halides to aldehydes or ketones, and amines to subunits ${ }^{[27]}$

同年, Sawant 等 ${ }^{[28]}$ 在 $\mathrm{pH}$ 值为 1 和 2 的水介质中, 对 Anderson 型钴(III)多酸盐阴离子 $\left[\mathrm{Co}^{\mathrm{III}}(\mathrm{OH})_{6} \mathrm{Mo}_{6} \mathrm{O}_{18}\right]^{3-}$ 催 化氧化乙酰氨基酚的反应进行了相关研究, 在该反应 中, 氧化剂和钴钼酸盐离子对反应的抑制表明各种形式 的氧化剂之间存在优先平衡, 反应开始从中性对乙酰氨 基酚到阴离子进行直接电子转移，随后在速率测定步骤 中生成自由基，自由基进一步氧化成 $N$-乙酰基醌亚胺 作为中间体, 再经水解得到对苯醌和乙酸. 实验结果表 明反应物之间先形成一种弱配合物促进了反应的进行.

2019 年, 魏哲宇和茹诗等 ${ }^{[29]}$ 报道了以氧气作为唯 一氧化剂, 利用无机配体负载的 $\mathrm{Cu}$-Anderson 型杂多酸 催化剂 $\left(\mathrm{NH}_{4}\right)_{4}\left[\mathrm{Cu}(\mathrm{OH})_{6} \mathrm{Mo}_{6} \mathrm{O}_{18}\right]$ 来促进醇的好氧氧化的 研究. 经典过渡金属配合物催化剂对醇的催化氧化需要 复杂的有机配体或硝基自由基作为辅助催化剂, 许多反 应更需要用到强氧化物作为氧化剂, 而许多有机物与这 些强氧化剂接触会发生剧烈的爆炸, 因此传统的醇的催 化氧化反应往往过程复杂、成本高昂, 实验条件严苛且 危险，而这种 $\mathrm{Cu}$-Anderson 型杂多酸催化剂由廉价的七 钿酸铵和硫酸铜在水中合成, 对于醇的催化氧化反应中 多种不同的底物也表现出优异的选择性和活性, 这种方 法不仅安全高效而且绿色环保, 更有希望实现工业化应 用. 实验结果表明, 该催化体系的催化机理如下(图 7 ${ }^{[29]}$ : 对于无机配体负载的铜催化剂 $\left(\mathrm{NH}_{4}\right)_{4}\left[\mathrm{Cu}(\mathrm{OH})_{6}-\right.$ $\mathrm{Mo}_{6} \mathrm{O}_{18}$ ], 氧化所需的两个氧化当量不仅存储在铜中心, 而且可以转移到 6 个边缘化的 $\mathrm{MoO}_{6}$ 单元上. $\mathrm{Cu}$-Anderson 型杂多酸催化剂与乙醇反应生成活性物质 $\mathrm{A}$, 而 $\mathrm{A}$ 和 $\mathrm{E}$ 是一对可以相互转换的异构体, 这可能是 因为电子通过分子内氧桥 $\mathrm{Cu}-\mathrm{O}-\mathrm{Mo}$ 转移到无机配体 $\mathrm{MoO}_{6}$ 上, 从而导致其中一个 $\mathrm{Mo}$ 单元从正六价变为正 五价. Cu-Anderson 型杂多酸 A 首先激活分子氧生成活 性物种 $B$. 随后, 物种 $B$ 经过 $O=O$ 键杂化得到具有高 活性的金属氧物种 $\mathrm{C}$ 作为活性氧化剂. 在这个催化反应 过程中, 通过 MS 检测表明水参与了产生过氧化氢的反 应, 因此该催化体系更接近于半乳糖氧化酶反应, 其中 $\mathrm{Cu}^{\mathrm{II}}$ 和羟基自由基作为一个单电子氧化剂共同作用, 介 导一个双电子醇氧化反应. 在半乳糖氧化酶中反应, 一 个单一的铜中心与氧气反应, 产生过氧化氢作为副产
物. 高活性的物种 $\mathrm{C}$ 与乙醇反应生成中间体 $\mathrm{D}$, 然后提 取 $\mathrm{H}$ 原子生成醛并再生 $\mathrm{E}$. 最后, 中间体 $\mathrm{E}\left(\mathrm{Cu}^{\mathrm{II}}\right)$ 被过氧 化氢氧化回物质 $\mathrm{A}\left(\mathrm{Cu}^{\mathrm{I}}\right)$.

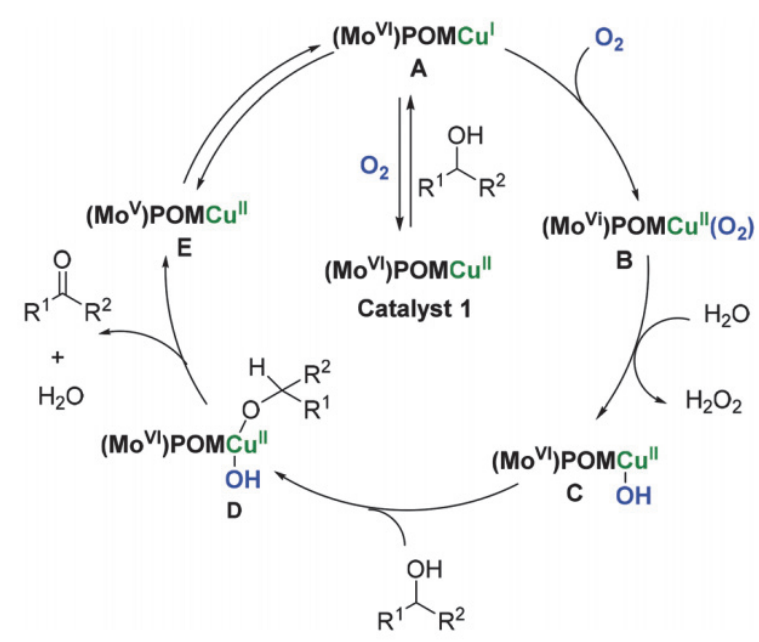

图 $7\left(\mathrm{NH}_{4}\right)_{4}\left[\mathrm{Cu}(\mathrm{OH})_{6} \mathrm{Mo}_{6} \mathrm{O}_{18}\right]$ 催化醇氧化的机理研究 ${ }^{[29]}$

Figure 7 The catalytic mechanism of alcohol oxidation by using $\left(\mathrm{NH}_{4}\right)_{4}\left[\mathrm{Cu}(\mathrm{OH})_{6} \mathrm{Mo}_{6} \mathrm{O}_{18}\right]^{[29]}$

同年，戴国勇和余焓等 ${ }^{\left[{ }^{[0]}\right.}$ 报道了以 $30 \% \mathrm{H}_{2} \mathrm{O}_{2}$ 为氧 化剂, 利用无机配体负载 Fe-Anderson 型杂多酸催化剂 $\left(\mathrm{NH}_{4}\right)_{3}\left[\mathrm{Fe}(\mathrm{OH})_{6} \mathrm{Mo}_{6} \mathrm{O}_{18}\right.$ ]催化烯烃环氧化反应的研究，该 反应不需要额外的还原剂或自由基作引发剂, 且实验操 作简单，完全避免了使用昂贵、有毒的贵金属催化剂， 不需要使用对空气/水分敏感且商业上不可用的有机配 体或铇多酸等. 相关催化剂 $\left(\mathrm{NH}_{4}\right)_{3}\left[\mathrm{Fe}(\mathrm{OH})_{6} \mathrm{Mo}_{6} \mathrm{O}_{18}\right]$ 在 $100{ }^{\circ} \mathrm{C}$ 的水中一步反应合成制备, 其催化机理与铁卟啉 型类似。该催化剂成功地将各种芳香族和脂肪族烯烃转 化成相应的环氧化合物, 具有良好的产率和选择性.

余焓、王静静等 ${ }^{[31]}$ 进一步利用 Fe-Anderson 型杂多 酸 $\left(\mathrm{NH}_{4}\right)_{3}\left[\mathrm{Fe}(\mathrm{OH})_{6} \mathrm{Mo}_{6} \mathrm{O}_{18}\right]$ 在温和的条件下实现了各种 醛与醇的氧化酯化反应并获得了高产率的酯类化合物, 包括几种药物分子和天然产物等. 吴志康、魏哲宇和赵 启新等 ${ }^{[32]}$ 利用无机配体负载的 Cr-Anderson 型杂多酸催 化剂 $\left(\mathrm{NH}_{4}\right)_{3}\left[\mathrm{CrMo}_{6} \mathrm{O}_{18}(\mathrm{OH})_{6}\right]$ 在温和的条件下, 以甲醇作 为潜在的甲酰化试剂, 成功地将各种伯胺和仲胺转化成 相应的甲酰胺, 并对二元伯胺进行了甲酰化反应. 该低 价铬催化剂相较于有机合成中广泛使用的高价铬催化 剂(包括 $\mathrm{CrO}_{3}$ 和 $\mathrm{K}_{2} \mathrm{Cr}_{2} \mathrm{O}_{7}$ 等)不仅更有效, 而且安全、绿 色环保. 吴志康等 ${ }^{[33]}$ 又以无机配体负载的 Fe-Anderson 型杂多酸催化剂 $\left(\mathrm{NH}_{4}\right)_{3}\left[\mathrm{Fe}(\mathrm{OH})_{6} \mathrm{Mo}_{6} \mathrm{O}_{18}\right]$ 催化甲酸和胺 偶联生成甲酰胺。徐佳佳等 ${ }^{[34]}$ 开发了一种基于 Fe-Anderson 型多金属氧酸盐 $\mathrm{Na}_{3} \mathrm{Fe}(\mathrm{OH})_{6} \mathrm{Mo}_{6} \mathrm{O}_{18} \cdot 5 \mathrm{H}_{2} \mathrm{O}$ 选择性氧化 5-差弪甲基糠醛制备 5-甲酰-2-呋喃甲酸的新 方法, 实验表明该催化剂在常压好氧环境下有较高的催 化活性. 
2020 年王静静、余焓和魏哲宇等 ${ }^{[35}$ 提出并证实了 一种无机配体负载的单钴离子催化剂 Co-Anderson 型杂 多酸催化剂 $\left(\mathrm{NH}_{4}\right)_{3}\left[\mathrm{Co}(\mathrm{OH})_{6} \mathrm{Mo}_{6} \mathrm{O}_{18}\right]$ 的催化氧化反应. 在该催化剂存在下, 以 $\mathrm{KCl}$ 为添加剂可以有效地促进 $30 \% \mathrm{H}_{2} \mathrm{O}_{2}$ 直接氧化醇制备酯类. 酯作为生物、医药、精 细化工等领域中一类重要的合成因子, 通常由羧酸或活 性衍生物(酰氯和酸䣶)与醇反应制备. 传统的醇转化为 酯的催化反应步骤繁琐, 反应条件严苛, 副产物较多且 底物适应性有限, 而王静静等提出的这种廉价、稳定并 且在温和条件下能够安全高效绿色催化醇到酯的催化 体系具有非常重要的意义, 他们首先探索了苄醇和脂肪 醇与甲醇氧化偶联生成甲酯的底物范围, 结果表明各种 取代苄醇以及脂肪醇都能高效、高选择性地进行氧化酯 化反应, 最高产率可以达到 $99 \%$, 随后在苄醇和其衍生 物以及脂肪醇与甲醇成功氧化酯化的基础上, 王静静等 进一步将催化体系推广到茮醇以及脂肪醇与其它醇类 的酯化反应中, 均能高效地获得相应的酯类产物, 更重 要的是天然产物土木香内酯也可以通过此方法制备, 并 且产率可以达到 $66 \%$. 在该催化体系中, 在添加剂氯化 钾的作用下, 实验表明添加剂可以显著提升催化反应体 系的选择性和活性. 他们通过单晶结构衍射与解析发现 添加剂中的氯离子能够与催化剂 $\mathrm{CoMo}_{6}$ 在晶态时形成 超分子二聚体 $2\left(\mathrm{CoMo}_{6} \cdot \mathrm{Cl}\right)$ (图 8) 作为催化关键中间体, 然后该中间体与醇进一步经亲核加成和 $\beta$ 消除两步反应 实现了醇到酯的转化. 几乎在所有的情况下, 该催化剂 都能以较高催化活性使各种醇(芳香族和脂肪族)的氧化 交叉酯化在温和的条件下进行并得到相应的酯且产率 较高. 这项工作表明 Anderson 型多金属氧酸盐作为一 类重要的单金属离子无机分子载体, 是一种性能优异的 环境友好型催化剂, 在催化有机合成反应中具有很大的

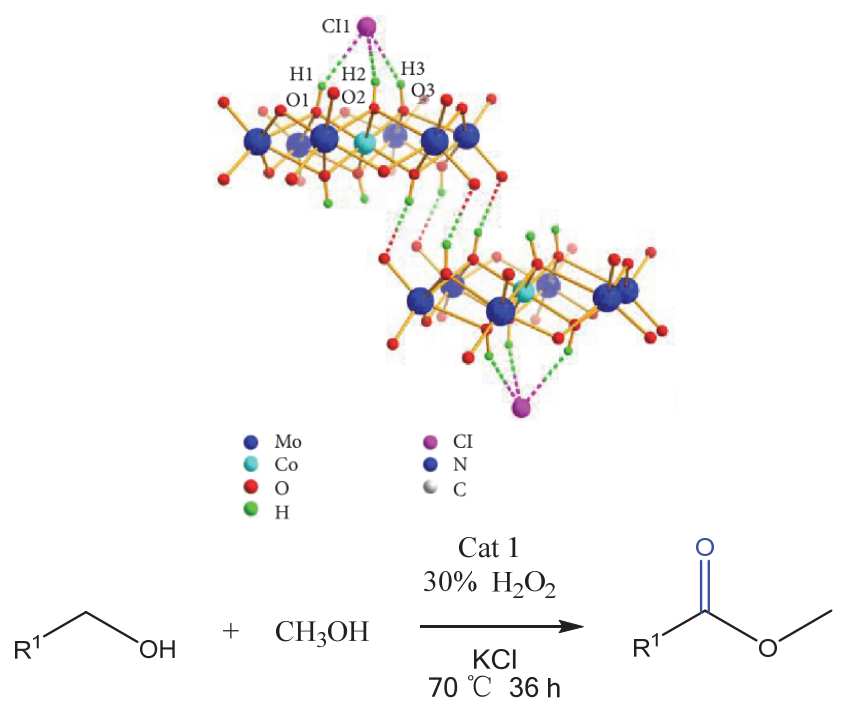

图 8 二聚体 $2\left(\mathrm{CoMo}_{6}{ }^{\circ} \mathrm{Cl}\right)$ 的超分子结构及催化路线 ${ }^{[35]}$

Figure 8 The supramolecular structure of dimer $2\left(\mathrm{CoMo}_{6} \cdot \mathrm{Cl}\right)$ and its catalytic route ${ }^{[35]}$
潜在应用价值. 其作为一种多功能的催化剂, 通过替换 Anderson 型多金属氧酸盐的中心金属, 改变其结构与性 质, 使得 Anderson 型杂多酸在醇的绿色催化氧化中显 示出优异的催化活性和极高的催化效率.

\subsection{Anderson 型杂多酸有机衍生物(PO)的催化}

\subsection{1 $[\mathrm{I}]^{+} \mathrm{PO}$}

2017 年, 魏永革课题组和罗建辉等 ${ }^{[36]}$ 报道了一种 有机配体稳定的 $\beta$-异构体 Anderson 型锰钼酸盐亚纳米 团簇阴离子: $\left[\mathrm{NH}_{4}\right] \beta-\left\{\left[\mathrm{H}_{3} \mathrm{NC}\left(\mathrm{CH}_{2} \mathrm{O}\right)_{3}\right]_{2} \mathrm{MnMo}_{6} \mathrm{O}_{18}\right\}$. 该 亚纳米团簇作为一种高活性的催化剂, 可以选择性催化 环己酮、环己醇或其混合物 KA 油的氧化(图 9). 研究结 果表明不仅 Anderson 型杂多酸特殊的蝶形拓扑结构对 催化活性有显著的提高, 而且该催化剂的高选择性和高 催化转化效率可以使其被用于己二酸的绿色工业化合 成.

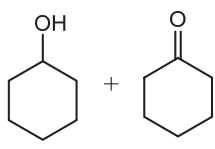

KA oil

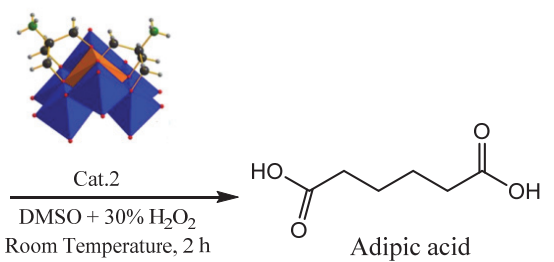

$93 \%$ yield , >99.9\% conversion
图 $9 \beta-\left\{\left[\mathrm{H}_{3} \mathrm{NC}\left(\mathrm{CH}_{2} \mathrm{O}\right)_{3}\right]_{2} \mathrm{MnMo}_{6} \mathrm{O}_{18}\right\}$ 催化 $\mathrm{KA}$ 油一锅选择性氧化制 取已二酸 ${ }^{[36]}$

Figure $9 \quad \beta-\left\{\left[\mathrm{H}_{3} \mathrm{NC}\left(\mathrm{CH}_{2} \mathrm{O}\right)_{3}\right]_{2} \mathrm{MnMo}_{6} \mathrm{O}_{18}\right\}^{-}$catalyzed selective oxidation of KA oil to adipic acid in one pot ${ }^{[36]}$

\subsection{2 $[\mathrm{O}]^{+} \mathrm{PO}$}

2017 年，余焓等 ${ }^{[37]}$ 开发了第一个用有机杂化 $\mathrm{Fe}-$ Anderson 型杂多酸催化剂 $\left[\mathrm{N}\left(\mathrm{C}_{4} \mathrm{H}_{9}\right)_{4}\right]_{3}\left[\mathrm{FeMo}_{6} \mathrm{O}_{18}\right.$ $(\mathrm{OH})_{3}\left\{\left(\mathrm{OCH}_{2}\right)_{3} \mathrm{CNH}_{2}\right\}$ ] 催化水中醛的好氧氧化反应(图 $10)$, 催化研究开始于在水溶液反应条件下, Fe-Anderson 型杂多酸作为催化剂 $(0.1 \mathrm{~mol} \%)$, 用 $\mathrm{O}_{2}\left(1.013 \times 10^{5} \mathrm{~Pa}\right)$ 氧化苯甲醛生成苯甲酸. 他们用不同的添加剂在 $50{ }^{\circ} \mathrm{C}$ 时进行了反应，发现添加剂的碱度对产物转化率有着很 大的影响. 在该催化反应体系中加入 $\mathrm{Na}_{2} \mathrm{CO}_{3}$ 时 $\left(\mathrm{p} K_{\mathrm{b}}=\right.$ $3.67)$, 苯甲酸的产率为 $95 \%$, 而加入 $\mathrm{NaHCO}_{3}$ 时 $\left(\mathrm{p} K_{\mathrm{b}}=\right.$ $7.95)$, 苯甲酸的产率降为 $63 \%$. 加入 $\mathrm{Na}_{2} \mathrm{SO}_{3}\left(\mathrm{p} K_{\mathrm{b}}=6.8\right)$ 使产率进一步降低到 $26 \%$, 而加入 $\mathrm{Na}_{2} \mathrm{SO}_{4}\left(\mathrm{p} K_{\mathrm{b}}=12.0\right)$ 的产率最低为 $5 \%$. 对于有机碱性添加剂, 如 $\mathrm{CH}_{3} \mathrm{COONa}$ 和 $\mathrm{Et}_{3} \mathrm{~N}$, 得到苯甲酸的产率分别为 $83 \%$ 和 $89 \%$. 含中性盐的添加剂, 如 $\mathrm{NaBr} 、 \mathrm{KCl}$ 和 $\mathrm{NaCl}$, 只能 得到中等产率的氧化产物. 酸性添加剂 $\mathrm{NH}_{4} \mathrm{Cl}$ 对该催化 体系中催化氧化的过程有着明显的抑制作用. 这些结果 表明，添加剂对 Anderson 型多金属氧酸盐 $\mathrm{Fe}^{\mathrm{III}} \mathrm{Mo}_{6}$ 催化 剂的活性有着明显的影响，这或许是因为杂多酸的酸碱 性质具有很高的可调性. 随后, 他们还研究了催化剂用 量和反应温度对该催化反应的影响. 改变催化剂用量对 产物的收率影响不大，而随着反应温度的升高，苯甲酸 
的产率在 $50{ }^{\circ} \mathrm{C}$ 时达到最大值. 进一步将反应温度提高 到 $70{ }^{\circ} \mathrm{C}$ 时, 产率则降低到 $96 \%$, 这可能是由于氧气(气 相)与催化剂之间的接触减少所致. 在反应过程中, 从 通入氧气切换到在空气中进行反应, 在相同的大气压 下, 氧化产物的收率也可以达到 $90 \%$. 当在氮气球下进 行反应时, 获得产物的产率特别低, 仅为 $<5 \%$. 作为对 照实验, 在没有 $\mathrm{Fe}^{\mathrm{III}} \mathrm{Mo}_{6}$ 催化剂的情况下进行该催化反 应时, 只能检测到少量的产物生成. 余焓等提出的这种 Fe-Anderson 型杂多酸催化方法以氧气为唯一氧化剂, 在极温和的水溶液条件下进行, 并涵盖了多种官能团 醛. 此方法对环境友好、成本低廉且催化效益颇高, 具 有工业化应用价值.

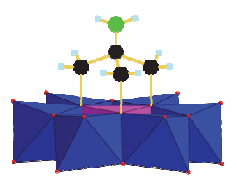

$\left[\mathrm{N}\left(\mathrm{C}_{4} \mathrm{H}_{9}\right)_{4}\right]_{3}\left[\mathrm{FeMo}_{6} \mathrm{O}_{18}(\mathrm{OH})_{3}\left\{\left(\mathrm{OCH}_{2}\right)_{3} \mathrm{CNH}_{2}\right\}\right]$

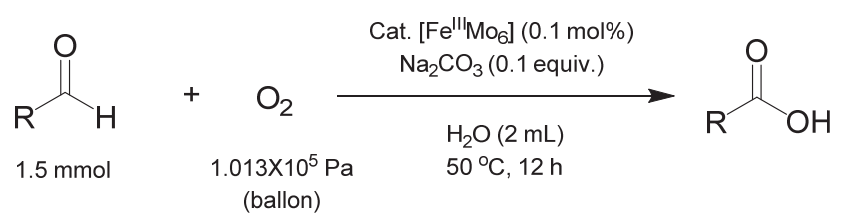

图 10 催化剂阴离子结构图及其催化反应路线 ${ }^{[37]}$

Figure 10 Catalyst anion structure and the catalytic reaction route ${ }^{[37]}$

余珊和殷盼超等 ${ }^{[38]}$ 发现卤素离子 $\left(\mathrm{X}^{-}\right)$可以在溶液 中结合在三羟甲基乙烷修饰的亚纳米级 Al-Anderson 型 多金属氧酸盐 $\left(\left[\left(n-\mathrm{C}_{4} \mathrm{H}_{9}\right)_{4} \mathrm{~N}\right]_{3}\left\{\mathrm{AlMo}_{6} \mathrm{O}_{18}(\mathrm{OH})_{3}\left[\left(\mathrm{OCH}_{2}\right)_{3}-\right.\right.\right.$ $\left.\left.\mathrm{CCH}_{3}\right]\right\}$ ) 的表面, 形成结合常数 $K=1.53 \times 10^{3}$ 的稳定超 分子复合物, 从而进行催化醇氧化到醛的反应(图 11). 晶体结构分析表明, 这种结合行为是通过卤素离子 $\mathrm{X}^{-}$ 和 $\left\{\mathrm{AlMo}_{6} \mathrm{O}_{18}(\mathrm{OH})_{3}\left[\left(\mathrm{OCH}_{2}\right)_{3} \mathrm{CCH}_{3}\right]\right\}^{3-}$ 的未修饰面上的三 个羟基之间形成多个 $\mathrm{X} \cdot \cdot \mathrm{H}-\mathrm{O}$ 氢键发生的. 这个超分 子亚纳米团簇体系中的超分子相互作用意味着其对于 从醇氧化到醛的催化活性可以在卤素离子和水的引入 下发生改变. 氯离子通过阻断团簇的活性中心而起到抑

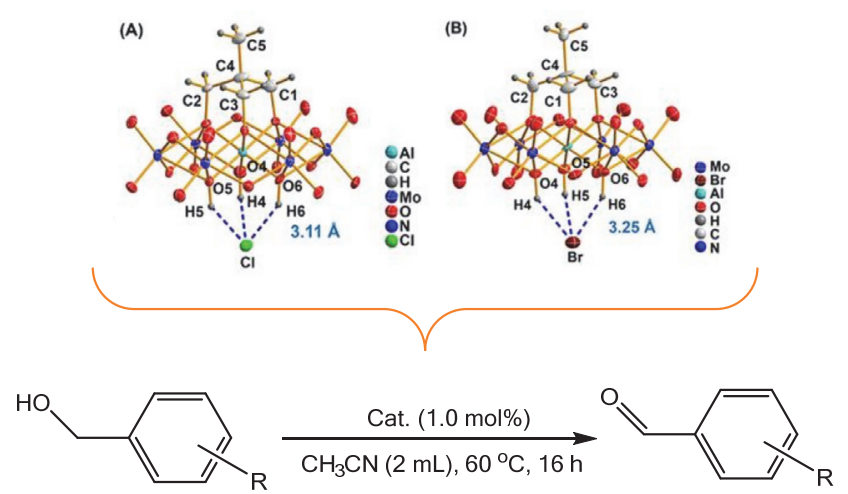

图 $11\left\{\mathrm{AlMo}_{6} \mathrm{O}_{18}(\mathrm{OH})_{3}\left[\left(\mathrm{OCH}_{2}\right)_{3} \mathrm{CCH}_{3}\right]\right\}^{3-} \cdot \mathrm{X}$ 催化氧化醇制醛 ${ }^{[38]}$

Figure $11\left\{\mathrm{AlMo}_{6} \mathrm{O}_{18}(\mathrm{OH})_{3}\left[\left(\mathrm{OCH}_{2}\right)_{3} \mathrm{CCH}_{3}\right]\right\}^{3-} \cdot \mathrm{X}$ catalytic oxidation of alcohols to aldehydes ${ }^{[38]}$
制作用，而团簇的催化活性可以通过加入水超分子取代 氯离子后被重新激活. 研究表明, 多重协同氢键的存在 是克服卤素离子与 Al-Anderson 型杂多酸阴离子之间静 电斥力的关键，卤素离子结合可调节 Al-Anderson 型杂 多酸的催化活性. 这项工作不仅丰富了 Anderson 型杂 多酸的超分子化学，同时也对催化氧化反应中催化剂的 选择性调控开辟了新的思路.

\section{3 总结与展望}

为解决传统化工生产所带来的环境危机, 自 20 世 纪 90 年代起, 对绿色化工技术的研究和开发逐渐成为 化学研究中的热门领域之一. 利用化学的技术和方法来 减少或消灭对人类健康和生态环境有害的物质产生是 绿色化工中的研究重点之一.

化工技术的进步和革新往往由新的催化材料和新 的催化技术来推动, 在催化领域的研究之中, Anderson 型多金属氧酸盐作为一类新型高效、绿色环保、廉价安 全的催化剂, 与其它无机酸相比, Anderson 型杂多酸本 身具有优异的氧化还原活性，同时它具有可测定并可控 的酸性, 在催化反应中有良好的双功能特性, 大多数多 酸阴离子溶解性很好, 不但具有确定的结构和尺寸, 而 且可以对其进行无机-有机修饰, 从而赋予其更加优异 的特性, 另外, 以 Anderson 型杂多酸作为催化剂不仅选 择性较高, 而且副反应少, 对设备几乎不腐蚀. 因此, Anderson 型杂多酸的催化应用在科学探索和绿色化工 技术方面有着巨大的应用前景和研究价值.

随着研究者们对于 Anderson 型杂多酸的深入探究, Anderson 型多金属氧酸盐及其衍生物在催化有机合成 反应中的应用对于绿色催化过程的研究逐渐表现出非 常重要的意义，此类杂多酸的催化应用正在被写入多酸 化学和合成化学的历史之中. 魏永革、韩生和余焓等课 题组更是对 Anderson 型杂多酸的结构调控及其在有机 合成反应中的催化应用研究做出了非常多的贡献.

结合相关的文献报道来看，简单的 Anderson 型杂 多酸被广泛应用于有机合成反应中，较为复杂的 Anderson 型杂多酸有机衍生物则可以针对特定的催化 反应来进行相关的多酸阴离子结构修饰，从而使催化剂 获得更好的催化特性. 以无机配体负载的 Anderson 型 多金属氧酸盐除了在醇的绿色催化氧化过程中展现出 优异的催化活性, 同时在催化 $\mathrm{C}-\mathrm{N}$ 键、 $\mathrm{C}-\mathrm{O}$ 键和 $\mathrm{C}-\mathrm{C}$ 键的形成也具有极高的效率. 相较于传统的贵金 属催化剂, Anderson 构型的多金属氧酸盐不仅合成原料 价格低廉，制备过程简单，催化剂的催化活性高，催化 反应过程也更加的绿色环保. 可以预期此类催化剂还将 在烃类的选择性氧化中起到重要的作用, 在工业催化领 域里具有非常广泛的应用前景和极高的工业价值.

由于一些 Anderson 型杂多酸催化剂在有机催化反 应往往表现出一定的不稳定性, 因此在实际催化过程 
里, 常常需要对不稳定的 Anderson 型杂多酸进行一系 列的有机-无机杂化修饰, 从而使其结构更加稳定, 利 于催化反应的进行.

本综述期望可以引起广大学者们对于 Anderson 型 杂多酸在有机催化领域的研究兴趣, 为更多的研究者们 提供一些新的方向和思路. 相信在不久的将来, 越来越 多的绿色环保的 Anderson 型杂多酸功能化材料将被应 用于更加广泛的有机合成反应中. Anderson 型杂多酸在 催化有机反应中的应用不仅会推动有机合成化学的发 展，更有可能开辟出一个全新的绿色催化合成世界.

\section{作者简介}

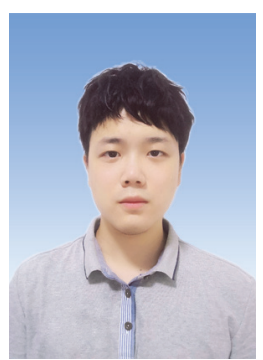

魏哲宇, 上海应用技术大学化学与环境工程学院应用化 学专业 2018 级硕士研究生, 目前致力于多酸化学领域的研究, 探索多酸的合成、结构、修饰及其催化应用.

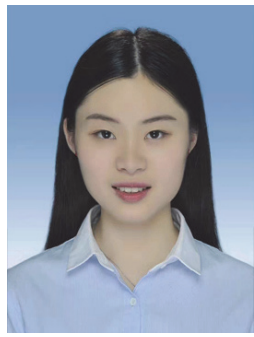

常亚林, 1997 年生于河南省幞阳市, 2019 年本科毕业于上 海应用技术大学化学与环境工程学院, 现为上海应用技术大 学化学与环境工程学院应用化学专业硕士研究生, 主要研究 方向为杂多酸的选择性催化氧化.

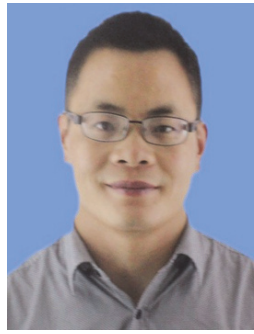

余焓博士, 清华大学博士后, 上海应用技术大学副教授. 先后承担国家自然科学基金、国家博士后基金、上海市联盟 计划企业委托开发等多个研究项目. 长期致力于有机合成及 不对称催化, 药物及其关键中间体的高效合成方法, 手性有 机无机杂化材料催化剂设计与合成及其应用等研究.

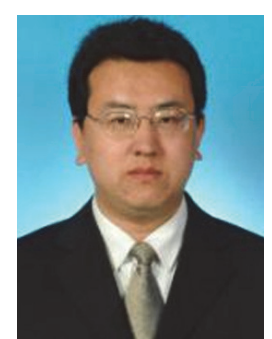

韩生博士，上海应用技术大学教授，博士生导师。长期从 事石油化工、精细化工等方面的研发工作。负责主持国家自然 基金面上项目、曙光计划、上海市人才发展基金、上海市科 委等各类课题四十余项. 《精细石油化工》特约编委, 废旧高 分子材料回收专家委员会委员、中国科学院青岛生物能源过 程工程所客座研究员等.

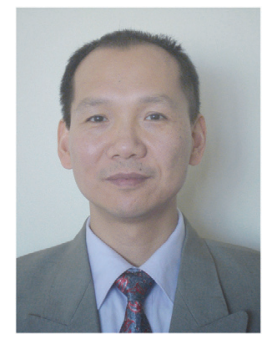

魏永革博士，清华大学教授、博士生导师，国家杰出青年 基金获得者. 当前的研究主要致力于多酸的合成、化学修饰和 多酸衍生物的反应化学与可控组装及其在有机合成、材料、 能源、环境和生物领域中的催化应用研究.

\section{References}

[1] Chen, W. L.; Wang, E. B. Polyoxometalate Chemistry, Science Press, Beijing, 2013, p. 38.

[2] Wang, N. J. Changchun Normal Univ. 2015, 34, 58.

[3] Wang, F. Ph.D. Dissertation, Northeast Normal University, Shenyang, 2007 (in Chinese). (王丰, 博士论文, 东北师范大学, 沈阳, 2007.)

[4] Qin, Z. X.; Li, Q.; Huang, Y. C.; Zhang, J. W.; Li, G.; Wei, Y. G. Chin. Sci. Bull. 2018, 63, 3263.

[5] Zhang, J. W.; Huang, Y. C.; Hao, J.; Wei, Y. G. Inorg. Chem. Front. 2017, 4, 1215.

[6] Wei, Z. Y.; Wei, Y. G. CN 104152980A, 2014 [Chem. Abstr. 2014, 9, $60]$.

[7] Hu, C. W.; Zhen, H.; Xu, L.; Wang, E. B. J. Mol. Sci. 1997, (1), 45 (in Chinese). (胡长文，甄慧，许林，王恩波，分子科学学报, 1997, $(1), 45$.

[8] Wu, P. S.; Zhang, H. Y.; Xu, L; De, G. J. R. H.; Hu, C. W.; Wang, E. B. J. Northeast Normal Univ. (Nat. Sci.) 2001, 4, 51 (in Chinese). (巫平松, 张宏宇, 许林, 德格吉日呼, 胡长文, 王恩波, 东北师 大学报(自然科学版), 2001, 4, 51.)

[9] Guo, S. R.; Kong, Y. M.; Peng, J.; Wang, E. B. Chem. Bull. 2007, 10,748 (in Chinese). (郭树荣, 孔育梅, 彭军, 王恩波, 化学通报, 2007, 10, 748.)

[10] Wei, Y. G. New progress of Polyacid Imine Derivatives, Abstracts of the 6th annual academic meeting and member congress of China Crystal Society (functional molecular crystal branch), China Crystal Society, 2016, p. 28.

[11] Wei, Z. Y.; Li, Q.; Wei, Y. G. J. Mol. Sci. 2017, 33, 391 (in Chinese). (魏哲宇, 李琦, 魏永革, 分子科学学报, 2017, 33, 391.)

[12] Song, Y. F.;Wei, Y. G. Chin. Sci. Bull. 2018, 63, 3261 (in Chinese) (宋宇飞，魏永革，科学通报，2018，63, 3261.)

[13] Yu, F. L.; Liu, C. Y.; Xie, P. H.; Yuan, B.; Xie, C. X.; Yu, S. T. RSC $A d v . \mathbf{2 0 1 5}, 5,85540$.

[14] Lei, Y.; Li, Z.; Yuan, Z.; Wang, R.; Sunee, W.; Dong, Z. L. Sep. Purif. Technol. 2015, 151, 155. 
[15] Li, P. C. M.S. Dissertation, Yantai University, Yantai, 2017 (in Chinese). (李鹏程, 硕士论文, 烟台大学, 烟台, 2017.)

[16] Yang, W.; Hou, Y. J.; An, H. Y. J. Mol. Sci. 2017, 33, 385 (in Chinese). (杨微, 侯玉姣, 安海艳, 分子科学学报, 2017, 33, 385.)

[17] Sun, L.; Su, T.; Li, P. Catal. Lett. 2019, 149, 7.

[18] Ji, H. B.; She, Y. B. Prog. Chem. Eng. 2007, 26, 605 (in Chinese). (纪红兵, 余远斌, 化工进展, 2007, 26, 605.)

[19] Li, J. J.; Wu, F. Textbook of introduction to green chemistry, Wuhan University Press, WuHan, 2015, p. 8.

[20] He, Y. M.; Sun, Y. H.; Han, B. X. Chin. Sci. Bull. 2015, 16, 1421.

[21] Song, J. L.; Han, B. X. Natl. Sci. Rev. 2015, 3, 255.

[22] SD, K.; Gokavi, G. S. Res. J. Chem. 2016, 6, 17.

[23] Yu, H.; Zhai, Y. Y.; Dai, G. Y.; Ru, S.; Han, S.; Wei, Y. G. Chem.-Eur. J. 2017, 23, 13883 .

[24] Yu, H.; Ru, S.; Zhai, Y. Y.; Dai, G. Y.; Han, S.; Wei, Y. G. Chem CatChem 2018, 10, 1253.

[25] Zhai, Y. Y.; Zhang, M. Q.; Fang, H. B.; Ru, S.; Yu, H.; Zhao, W. S.; Wei, Y. G. Org. Chem. Front. 2018, 5, 3454.

[26] Zhang, M. Q.; Zhai, Y. Y.; Ru, S.; Zang, D. J.; Han, S.; Yu, H.; Wei, Y. G. Chem. Commun. 2018, 54, 10164.

[27] Wang, J. J.; Zhai, Y. Y.; Wang, Y.; Yu, H.; Zhao, W. S.; Wei, Y. G. Dalton Trans. 2018, 47, 13323.
[28] Sawant, J. D.; Patil, K. K.; Gokavi, G. S. Transition Met. Chem. 2019, 44, 153.

[29] Wei, Z. Y.; Ru, S.; Zhao, Q. X.; Yu, H.; Zhang, G.; Wei, Y. G. Green Chem. 2019, 21, 4069.

[30] Zhou, Z. H.; Dai, G. Y.; Ru, S.; Yu, H.; Wei, Y. G. Dalton Trans. 2019, 48,14201 .

[31] Yu, H.; Wang, J. J.;Wu, Z. K.; Zhao, Q. X.; Dan, D. M.; Han, S.; Tang, J. J.; Wei, Y. G. Green Chem. 2019, 21, 4550.

[32] Yu, H.; Wu, Z. K.; Wei, Z. Y.; Zhai, Y. Y.; Ru, S.; Zhao, Q. X.; Wang, J. J.; Han, S.; Wei, Y. G. Commun. Chem. 2019, $2,1$.

[33] Wu, Z. K.; Zhai, Y. Y.; Zhao, W. S.; Wei, Z. Y.; Yu, H.; Han, S.; Wei, Y. G. Green Chem. 2020, 22, 737.

[34] Xu, J. J.; Zhu, Z. G.; Yuan, Z. L.; Su, T.; Zhao, Y. C.; Ren, W. Z.; Zhang, Z. H.; Lu, H. Y. J. Taiwan Inst. Chem. E. 2019, 104, 8.

[35] Wang, J. J.; Yu, H.; Wei, Z. Y.; Qi, L.; Xuan, W. M.; Wei, Y. G. Research, 2020, 1, 3875920.

[36] Luo, J. H.; Huang, Y. C.; Ding, B.; Wang, P. M.; Geng, X. F.; Zhang, J. W.; Wei, Y. G. Catalysts 2018, 8,121 .

[37] Yu, H.; Ru, S.; Dai, G. Y.; Zhai, Y. Y.; Lin, H. L.; Han, S.; Wei, Y. G. Angew. Chem. Int. Ed. 2017, 56, 3867.

[38] She, S.; Mu, L.; Li, Q.; Huang, Z. H.; Wei, Y. G.; Yin, P. C. ChemPlusChem 2019, 11, 84 . 